\title{
The effect of inrush transients on PV inverter's grid impedance measurement based on inter-harmonic injection
}

\author{
V. Ćuk ${ }^{1}$, S. Bhattacharyya ${ }^{1}$, J.F.G. Cobben ${ }^{1}$, W.L. Kling ${ }^{1}$, R.B. Timens ${ }^{2}$ and F.B.J. Leferink ${ }^{2}$ \\ ${ }^{1}$ Department of Electrical Engineering, Eindhoven University of Technology \\ PO Box 513, CR. 2.06, $5600 \mathrm{MB}$, Eindhoven, the Netherlands \\ Phone: +31402474637, Fax:+31402450735, e-mail: V.Cuk@tue.nl \\ ${ }^{2}$ Department of Electrical Engineering, University of Twente
}

\begin{abstract}
This paper addresses a cause for false tripping of photovoltaic inverters with anti-islanding protection based on impedance measurement with inter-harmonic injection. Earlier discussions about tripping problems happening when several devices are doing the measurement at the same time are supplemented with a problem caused by inrush transients of nearby devices.
\end{abstract}

A series of experiments was conducted in the Power Quality laboratory of the TU/e, on a PV inverter which complies with the DIN VDE 0126 standard. Impedance measurement was done in parallel with the inverter and measurement results are presented. A criterion for false tripping caused by transients is explored. Also, influences of network impedance and grid harmonic pollution on false tripping were analyzed. In the end, some signal processing techniques are proposed to avoid this problem.

\section{Key words}

Grid impedance measurement, transient, PV inverter, power quality, anti-islanding protection.

\section{Introduction}

Photovoltaic inverter manufacturers are obliged to meet power quality regulations for connecting inverters to the grid. Some technical regulations (for example German DIN VDE 0126 standard and European regulation EN 503301-1) have a requirement for the inverter's antiislanding protection. The anti-islanding protection has two main objectives. One is to prevent dangerous voltage presence in parts of the network disconnected from the rest of the grid for maintenance or other reasons. The other is to prevent the inverter to supply an islanded part of the network (together with other distributed generators) with voltage which doesn't meet local power quality requirements.

Numerous anti-islanding methods are developed by now. They are sorted and described with more details in [1]. Globally, they are distinguished as passive, active, and methods which are not resident in the inverter. Passive methods are non-invasive, and use only monitoring of voltage and frequency. Active methods deliberately inject disturbances into the grid, and then monitor its response. Methods not resident in the inverter are implemented in separate measurement devices controlled by the utility company.

This paper aims one of the active methods - the impedance measurement method based on inter-harmonic injection. Experiments with a PV inverter are done in which its anti-islanding protection was tripping falsely due to inrush transients. Results of these experiments are presented and discussed in the paper, and some proposals for measurement improvements are given.

\section{Impedance measurement method}

One of the widely used active methods for anti-islanding detection is impedance measurement. This method is suggested by DIN VDE 0126 and EN 50330-1 standards. Requirements for this type of protection is that it detects a change in the network impedance equal or higher than $0.5 \Omega$, and that it reacts within $5 \mathrm{~s}$. Impedance measurement can be done with power shifting or injection of non-characteristic harmonics or interharmonics. Injection of harmonics or inter-harmonics is a widely used variant of this method. It uses a deliberate disturbance which will produce a current at the desired frequency. Simultaneously, voltage and current are measured and their (inter)harmonic components are derived. Impedance is then calculated by dividing the values of voltage and current (and scaled to $50 \mathrm{~Hz}$ ). To reduce measurement errors, averaging of impedance results is often used.

One of the problems mutual for both variants of impedance measurement is inaccurate measurement caused by parallel operation of several devices [1]. In case of power shifting, impedance measurement suffers from lower sensitivity due to unsynchronized power shifts [1]. In case of harmonic or inter-harmonic injection, false tripping is possible because injection of harmonic currents of several devices will increase the overall harmonic voltage [1-4]. 
To reduce the erroneous effect caused by parallel injection, altered injection methods are developed to desynchronize the peaks of inter-harmonic currents $[2 ; 4]$. So it is possible to reduce the effect of deliberate disturbances, but the method is not always immune to uncontrolled disturbances, such as inrush transients. Their impact is not large when the voltage is not distorted, but when the voltage is distorted, higher and longer (inter)harmonic bursts are created during a transient. This was the motivation to explore their impact on impedance measurement.

\section{Laboratory setup}

A setup and a series of experiments on this were assembled in the Power Quality Laboratory of the TU/e, and it is schematically presented in Fig. 1. A commercially available inverter is fed from a photovoltaic simulator, made from a DC source and an array of diodes. The operating point is set by a maximum power tracking simulator. The AC side of the inverter is connected to the voltage source (California Instruments MX-45 programmable network simulator) which is controlled by a Matlab program. Between the network simulator and the inverter, a vacuum cleaner with electronic speed control is connected to produce intensive inrush transients. In front of the point of connection an impedance is placed to simulate network impedance. Its value is varied several times during the experiment.

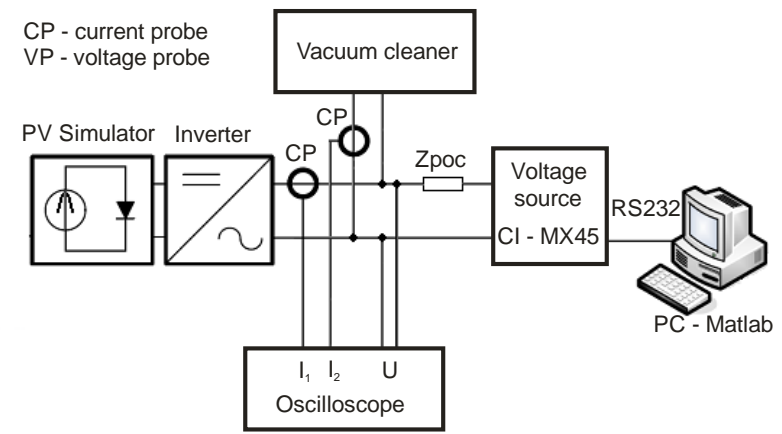

Fig. 1. Laboratory setup of the experiment

Voltage at the point of connection is measured by a Hioki 8861 oscilloscope and data logger. Currents of the inverter and the vacuum cleaner are measured by the same oscilloscope and Tektronix TCP303 current probes. Measurement results are later processed in Matlab.

\section{Criterion for inverter's false tripping}

Data analysis showed that the impedance measurement is done on the frequency of $75 \mathrm{~Hz}$. This will be presented in chapter 5. To analyze the criterion for inverter's false tripping, an equivalent circuit of the experiment is depicted in Fig. 2. Abbreviations used in this figure are:

- $I_{i i}$ is the inter-harmonic current injected by the inverter,

- $I_{i v c}$ is the inter-harmonic current injected by the vacuum cleaner during the transient,

- $I_{i t}$ is the total inter-harmonic current flowing into the network,
- $U_{i i}$ is the inter-harmonic voltage measured by the inverter,

- $Z_{p o c}$ is the network impedance up to the point of connection at $75 \mathrm{~Hz}$,

- $Z_{i n v}$ is the impedance of inverter's connection at $75 \mathrm{~Hz}$,

- $Z_{v c}$ is the impedance of vacuum cleaner's connection at $75 \mathrm{~Hz}$.

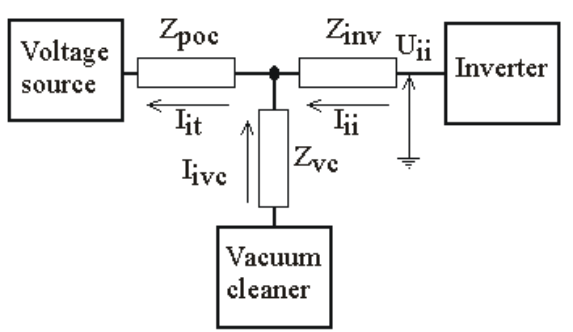

Fig. 2. Equivalent circuit of the experiment

The equivalent grid impedance value measured by the inverter (in this case at $75 \mathrm{~Hz}$ ) $Z_{M}$ can be calculated as:

$$
Z_{M}=\frac{U_{i i}}{I_{i i}}=\frac{Z_{p o c}\left(I_{i i}+I_{i v c}\right)+Z_{i n v} I_{i i}}{I_{i i}}
$$

The error in impedance measurement caused by transient inter-harmonics $Z_{\text {err }}$ is equal to:

$$
Z_{e r r}=\frac{Z_{p o c} I_{i v c}}{I_{i i}}
$$

To cause a false tripping situation (exceed the error of 0.5 $\Omega$ ), a transient has to satisfy the following condition:

$$
Z_{p o c} I_{i v c}=0.5 \cdot I_{i i}
$$

Condition (3) has to be met during a time interval needed for one or several measurements. Chapter 6 will further discuss the relation between the error magnitude and its time duration if averaging of consecutive impedance results is used. For the inverter analyzed, used value of $I_{i i}$ is between $1 \mathrm{~A}$ and $1.2 \mathrm{~A}$, which gives the values between $0.5 \mathrm{~V}$ and $0.6 \mathrm{~V}$ on the right hand side of equation (3).

Impact of the impedance $Z_{v c}$ on the measurement is not significant if it is a single branch. As the inter-harmonic source behaves like a current source, this impedance should not influence the measurement unless it is big enough to change the nature of the transient (so it doesn't behave like a current source). If it would include some shunt elements, it could absorb a part of the interharmonic current and reduce the erroneous effect.

The effect of the impedance $Z_{i n v}$ is more significant than the effect of $Z_{v c}$. If it has a value higher than $Z_{p o c}$, most of the voltage $U_{i i}$ will be composed of the voltage drop on the impedance $Z_{i n v}$, which will make the measurement less sensitive to the current flowing through the impedance $Z_{\text {poc }}$. That means that a higher value of $Z_{i n v}$ 
reduces sensitivity to transients (but it also reduces the accuracy of $Z_{\text {poc }}$ measurement).

\section{Measurement results}

The experiment was repeated several times in different conditions. Every inrush was starting while the inverter was operating connected to the network simulator in a steady state. Vacuum cleaner was being started in the position of its lowest speed, since then it generates more harmonics. Voltage from the network simulator was altered several times. The first time it was a clean sine wave, and then a few configurations of harmonics were added. Also, grid impedance was altered several times. In some situations the inverter would trip, while in other situations it would not. These conditions and occurrences of trips are given in Table I. Every measurement number (No) represents several repetitions with the same conditions.

Table I. - Experiment conditions and results

\begin{tabular}{|c|c|c|c|}
\hline No & $\begin{array}{c}Z_{p o c} \text { at } \\
75 \mathrm{~Hz}[\Omega]\end{array}$ & $\begin{array}{c}\text { Voltage } \\
\text { distortion }\end{array}$ & $\begin{array}{c}\text { tripping } \\
\text { occurrence }\end{array}$ \\
\hline 1 & $0.01+\mathrm{j} 0.005$ & several combinations & no trips \\
\hline 2 & $0.20+\mathrm{j} 0.010$ & $3 \% 5^{\text {th }}$ harmonic & no trips \\
\hline 3 & $0.20+\mathrm{j} 0.010$ & $5 \% 5^{\text {th }}$ harmonic & no trips \\
\hline 4 & $0.20+\mathrm{j} 0.010$ & $8 \% 5^{\text {th }}$ harmonic & 1 trip only \\
\hline 5 & $0.29+\mathrm{j} 0.480$ & $5 \% 5^{\text {th }}$ harmonic & 1 trip only \\
\hline 6 & $0.29+\mathrm{j} 0.480$ & $8 \% 5^{\text {th }}$ harmonic & every time \\
\hline 7 & $0.29+\mathrm{j} 0.480$ & $\begin{array}{l}5 \% 5^{\text {th }} \text { harmonic, } \\
5 \% 3^{\text {rd }} \text { harmonic }\end{array}$ & every time \\
\hline 8 & $0.09+\mathrm{j} 0.390$ & $\begin{array}{l}5 \% 5^{\text {th }} \text { harmonic, } \\
5 \% 3^{\text {rd }} \text { harmonic }\end{array}$ & every time \\
\hline 9 & $0.07+\mathrm{j} 0.215$ & $\begin{array}{l}5 \% 5^{\text {th }} \text { harmonic, } \\
5 \% 3^{\text {rd }} \text { harmonic }\end{array}$ & every time \\
\hline 10 & $0.07+\mathrm{j} 0.215$ & $\begin{array}{l}5 \% 5^{\text {th }} \text { harmonic, } \\
4 \% 3^{\text {rd }} \text { harmonic }\end{array}$ & every time \\
\hline 11 & $0.07+\mathrm{j} 0.215$ & $\begin{array}{l}4 \% 5^{\text {th }} \text { harmonic, } \\
3 \% 3^{\text {rd }} \text { harmonic }\end{array}$ & every time \\
\hline 12 & $0.07+\mathrm{j} 0.215$ & $\begin{array}{l}3 \% 5^{\text {th }} \text { harmonic, } \\
2 \% 3^{\text {rd }} \text { harmonic }\end{array}$ & no trips \\
\hline 13 & $0.09+\mathrm{j} 0.390$ & $\begin{array}{l}3 \% 5^{\text {th }} \text { harmonic, } \\
2 \% 3^{\text {rd }} \text { harmonic }\end{array}$ & no trips \\
\hline 14 & $0.40+\mathrm{j} 1.700$ & $\begin{array}{c}\text { no distortion \& } \\
\text { several distortion } \\
\text { combinations }\end{array}$ & $\begin{array}{l}\text { fault current } \\
\text { protection } \\
\text { trips }\end{array}$ \\
\hline
\end{tabular}

Impedance values used in experiments are chosen to cover most of the range which is usual in low voltage grids. In the Netherlands, impedance values in low voltage grids are almost always between $0.05 \Omega$ and 0.5 $\Omega$. In the modern part of the grid, the value of impedance is usually around $0.175 \Omega[5]$. In the measurement No 14 a very high value of impedance is used, to check the behavior in unusual conditions.

Vacuum cleaner's and inverter's current waveforms during an inrush and false tripping from measurement No 11 are presented in Fig. 3 and Fig. 4, respectively. The current spikes visible on the inverter's current waveform come in regular time intervals, and are caused by the inter-harmonic injection for impedance measurement. During normal operation (nothing detected by protections), impedance is measured on every two seconds. When something abnormal is measured by one of the protections, impedance measurement becomes more frequent (on every $200 \mathrm{~ms}$ ).

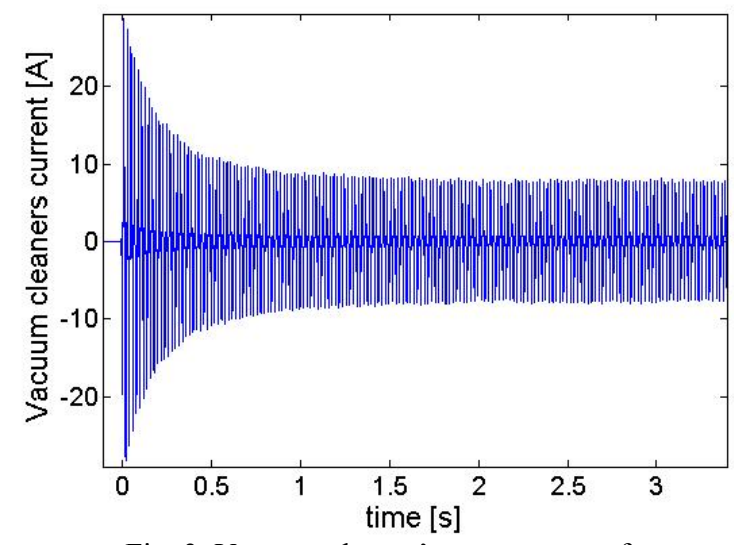

Fig. 3. Vacuum cleaner's current waveform

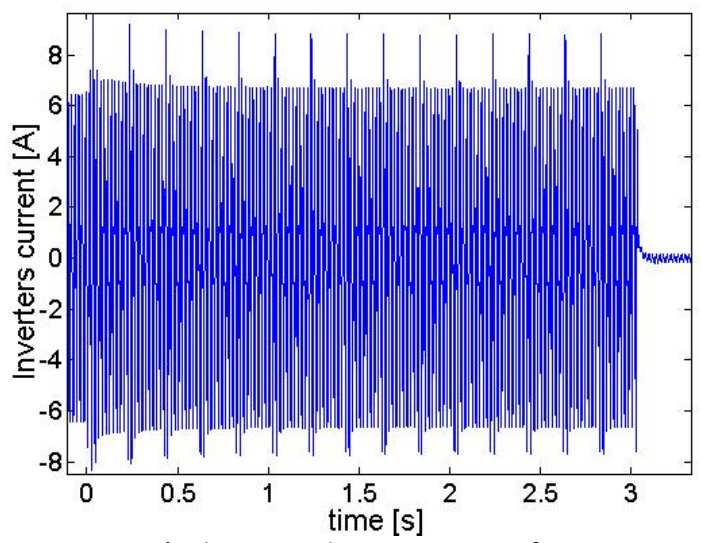

Fig 4. Inverter's current waveform

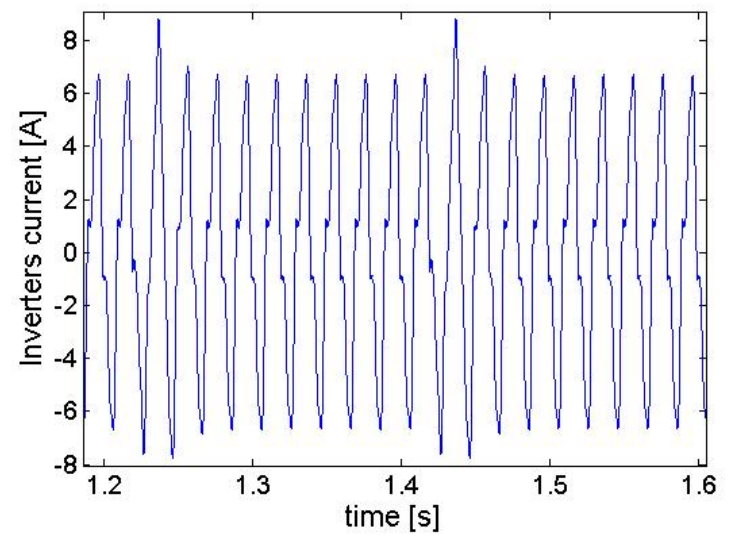

Fig. 5. Two inverter's injection cycles zoomed in

To analyze one injection cycle, Fig. 5 shows inverter's current zoomed in on a time interval of approximately two injection cycles $(400 \mathrm{~ms})$. One injection lasts two periods $(40 \mathrm{~ms})$, and is followed by an eight period pause. Injection duration of two periods implies that the resolution for inter-harmonic measurement is $25 \mathrm{~Hz}$. To have a higher resolution (less than $25 \mathrm{~Hz}$ ) more periods of injection are needed. To have a lower resolution (more than $25 \mathrm{~Hz}$ ) less periods of injection are needed, which would make a part of injection unnecessary.

To further explore the measurement frequency used in this anti-islanding protection, discrete Fourier transformation was done to inverter's current and voltage 
signals with different frequency resolutions. Resolutions used were: $16.67 \mathrm{~Hz}, 20 \mathrm{~Hz}, 25 \mathrm{~Hz}$ and $33.33 \mathrm{~Hz}$ (which correspond to data windows of 3, 2.5, 2 and 1.5 periods. All possible frequencies with those resolutions and up to $500 \mathrm{~Hz}$ were inspected. The frequency of $75 \mathrm{~Hz}$ had strongest current and voltage signals during injections, which makes it the most precise for impedance measurement. Further analysis of possible measurement frequencies was done using coherence functions for current and voltage data sets for one injection time interval [6]. Since the equivalent grid impedance is mostly linear in the frequency range observed, coherence values were very similar for all of the observed frequencies. Thus, coherence was excluded from further analysis. Based on the previous analysis, it was decided that $75 \mathrm{~Hz}$ is the measurement frequency used in the protection.

For the inrush and false trips analyzed, $75 \mathrm{~Hz}$ components of current and voltage are given in Fig. 6 and Fig. 7, respectively. The last peak from both figures should be neglected, as it presents the value at the moment when the inverter is being disconnected.

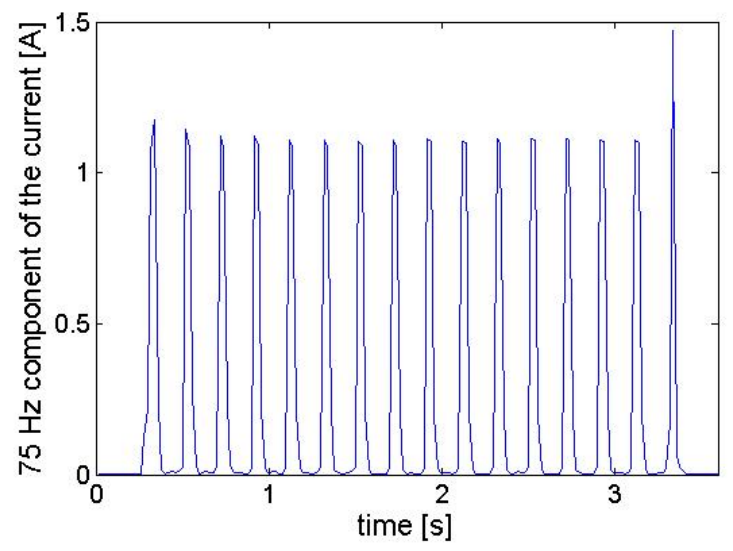

Fig. $6.75 \mathrm{~Hz}$ component of inverter's current

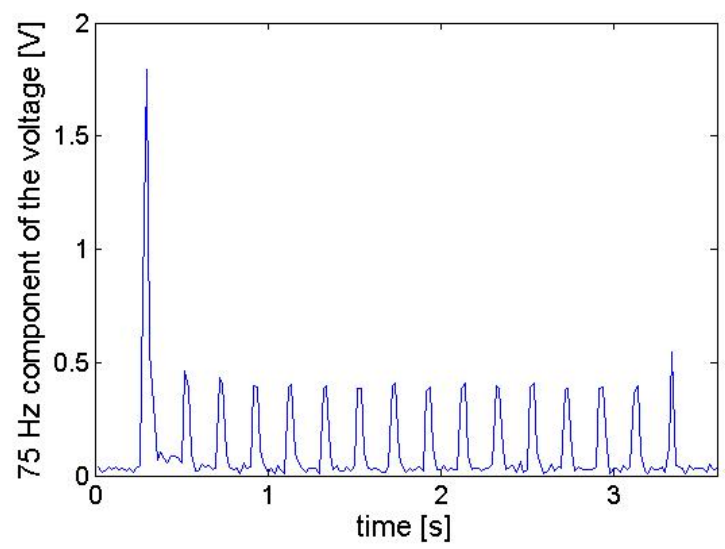

Fig. 7. $75 \mathrm{~Hz}$ component of inverter's voltage

The time change of impedance at $75 \mathrm{~Hz}$ calculated from these two time changes (calculated only at injection moments) is presented in Fig. 8. When the transient is settled down, calculated value of impedance is $0.36 \Omega$ in average. During the higher part of the transient it reaches a value of $1.67 \Omega$, which is approximately $1.4 \Omega$ higher than in normal conditions, and it is enough to trigger a false trip.

\section{Discussion on results}

\section{A. Influences of grid impedance and voltage distortion}

Looking at Table I, it is easy to understand the ways in which network impedance and voltage distortion influence the probability of false trip occurrence. As equation (3) suggests, higher values of network impedance require smaller values of transient interharmonic currents to produce a false trip. It can be seen in the table that with the smallest value of impedance (around $10 \mathrm{~m} \Omega$ ) no trips were caused. Starting from the next higher value (around $200 \mathrm{~m} \Omega$ ), trips could be caused. For the highest value of grid impedance used (around 1,750 $\mathrm{m} \Omega$, measurement No 14) behavior of anti-islanding protection couldn't be tested because the fault current protection was tripping at every inrush (this protection reacts much faster, within $100 \mathrm{~ms}$ ).

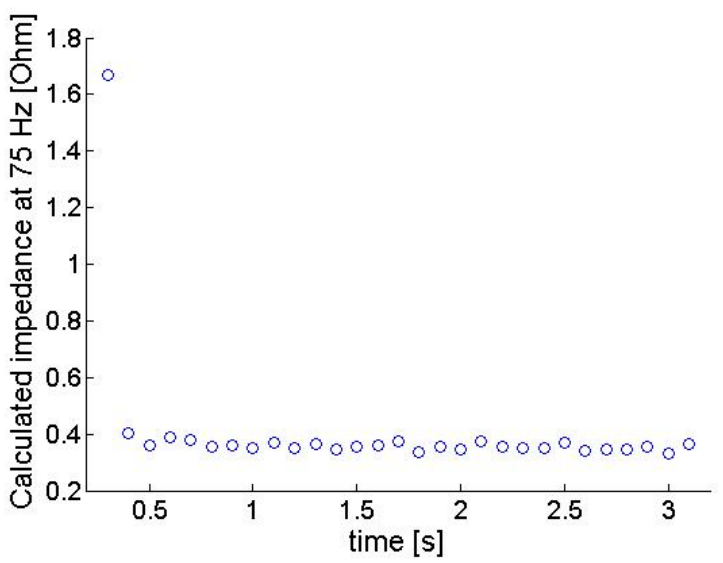

Fig. 8. Time change of calculated impedance at $75 \mathrm{~Hz}$

Also as expected, higher distortion levels were leading to higher probabilities of false tripping. Background distortion changes the shape of transients and by doing so increases the intensity of harmonic and inter-harmonic bursts. Table I clearly shows that effect. It is interesting to notice that a combination of two harmonics creates a greater effect than one harmonic with a high level. For example, the combination of $3 \%$ of the $3^{\text {rd }}$ harmonic and $4 \%$ of the $5^{\text {th }}$ harmonic (THD $=5 \%$ ), has a much stronger effect than just $5 \%$ of the $5^{\text {th }}$ harmonic (also $\mathrm{THD}=5 \%$ ), as seen in measurements No 5 and 11 from the table (measurement No 11 also has a lower $Z_{p o c}$ ).

In two situations (measurements No 4 and 5) false trips happened only one time (out of 10 tries). This shows that in those situations tripping occurs only if the transient starts at the "right" moment, when the measurement starts. Possibly it is also influenced by the starting voltage phase (since the transient intensity is dependent on the starting voltage phase). This indicates that a false trip could theoretically happen even in situations with smaller voltage distortion, if all timing coincidences are met. It also implies that a false trip doesn't have to happen every time with conditions labeled as "tripping every time" - measurements No 6 to 10 (in those cases the experiment was repeated less than 10 times per condition). 


\section{B. Influences of data window length and measurement frequency}

One way to reduce the influence of transients on impedance measurement is to increase the number of periods used for the discrete Fourier transformation (within the protection). Since the inrush inter-harmonic burst decays fast, shorter data windows can lead to calculation of larger voltage values, and thus the larger impedance values. For longer data windows, the maximum is dumped with smaller values that follow it. To illustrate this, Fig. 9 shows the $75 \mathrm{~Hz}$ current component of a vacuum cleaner starting at a voltage phase of 0 degrees, when the voltage has no harmonic distortion. DFT is done with three different data windows: 2,4 and 6 periods.

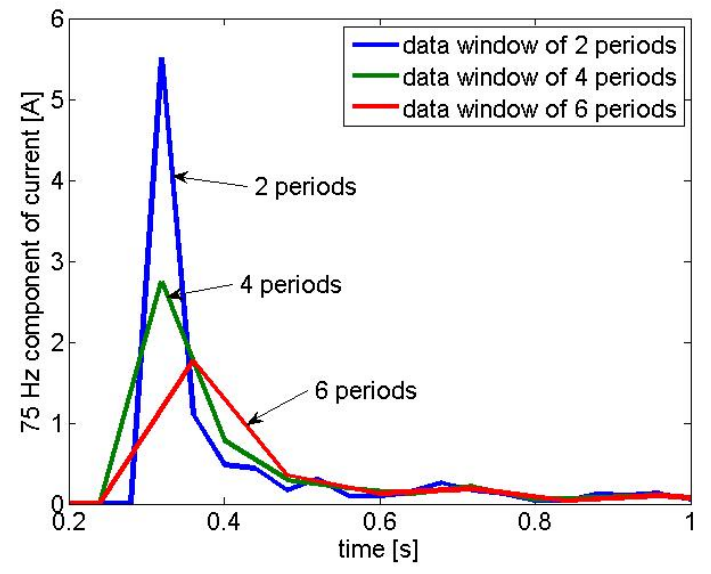

Fig. $9.75 \mathrm{~Hz}$ component of vacuum cleaner's starting current calculated with 2, 4 and 6 periods

For the measurement frequency of $75 \mathrm{~Hz}$, possible numbers of periods are multiples of 2 (2, 4, 6, etc.), because the required frequency resolution is $50 / 2 \mathrm{~Hz}$. For different frequencies (different frequency resolutions), different series of numbers of periods have to be used (e.g. for $500 / 3 \mathrm{~Hz}$ a multiple of 3 is needed, since the resolution needed is $50 / 3 \mathrm{~Hz}$ ).

Using a very long data window has a drawback of having too many periods with the disturbance, which makes a limitation for increasing it. More periods with the disturbance would increase the overall disturbance level, and that would ask for a smaller amount of interharmonic current injected to meet the disturbance limits. That would decrease the accuracy of measurement. So the optimal data window length is not very long, but it is bigger than 2 periods. In combination with averaging of the impedance value calculated, 3 or 4 periods might be sufficient without a big decrease of the inter-harmonic current injected.

When it comes to the selection of measurement frequency, three things have to be considered. The first restriction is the local bandwidth of ripple control signals. For example in the Netherlands, ripple control signals are placed on frequencies equal or higher than $210 \mathrm{~Hz}$. The second thing to be considered is the frequency dependence of the grid impedance. As it is almost monotonically increasing with frequency, it is not good to use a very high measurement frequency, because in that case small current or voltage measurement errors would lead to huge impedance calculation errors. The third thing that can be considered is the possible spectrum of frequencies present during the inrush transient burst. Most of the inrushes will have higher inter-harmonic values at lower frequencies, and their values decrease as the frequency increases. This is illustrated in Fig. 10, which shows the inrush time change of several inter-harmonic components for a vacuum cleaner when a clean sine wave is applied to it. It is noticeable that the peak of the $175 \mathrm{~Hz}$ component is about 9 times smaller than of the $25 \mathrm{~Hz}$ component. The peak of $25 \mathrm{~Hz}$ component is even higher than the one of $75 \mathrm{~Hz}$ component, which here has a peak which is almost identical to the peak of the $125 \mathrm{~Hz}$ component.

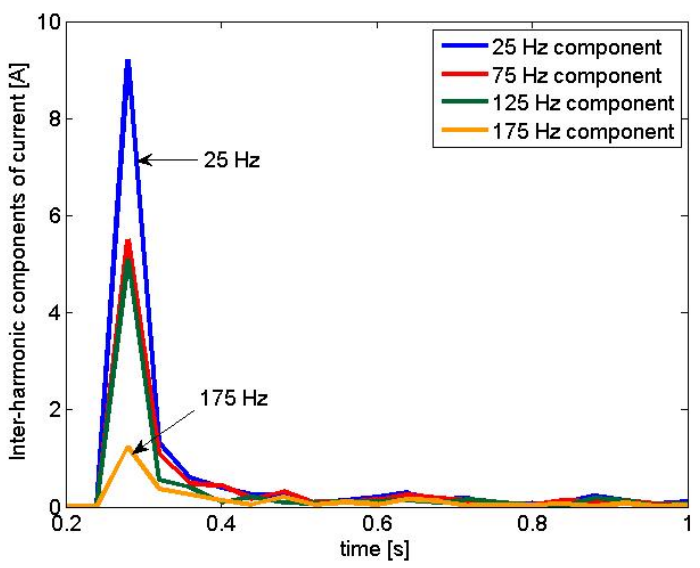

Fig. 10. Time changes of several inter-harmonic current components during a vacuum cleaner inrush

Having all of this in mind, the optimal measurement frequency should be higher than $150 \mathrm{~Hz}$ and sufficiently lower than the lowest ripple control frequency (to avoid interference). In the case of the Netherlands, the range would be $150 \mathrm{~Hz}-200 \mathrm{~Hz}$.

\section{Influence of result averaging}

If consecutive impedance values are averaged, probability of false tripping is reduced. In that case more erroneous values are needed (or higher error values) to make the end result higher for $0.5 \Omega$ or more. Minimal value of $I_{v c}$ and its duration above the limit given by (3) are in this case dependent on the number of values used for averaging and the time between consecutive measurements. The end result has an error which is equal to the sum of all errors divided by the number of measurements used for averaging $N$. Tripping will occur when the sum of all errors is greater than $0.5 \cdot N[\Omega]$, so the immunity can be increased if $N$ is made higher.

Another approach which can be used along with averaging is skipping measurement results in a short time interval after the first high measurement value (including the first value). If for example $0.5 \mathrm{~s}$ is skipped, most of transients will settle down and there is still sufficient time to react within $5 \mathrm{~s}$ from the moment of islanding if it appears at the same time. 


\section{Conclusions}

An analysis on the influence of inrush transients on the impedance measurement based on inter-harmonic injection is presented in the paper. Experiments showed that interference is possible if certain conditions are met:

- there is a source of inter-harmonic bursts,

- certain voltage distortion is present (in this case a combination of the $3^{\text {rd }}$ and $5^{\text {th }}$ harmonic had the biggest effect, while the distortion was still within the EN 50160 standard limits), and

- network impedance has at least a certain value which is dependent on the measurement injection current (in this case it was a value which is possible in low voltage networks, approximately $0.225 \Omega$ ).

Some other requirements can be explored, in terms of the sensitivity of these phenomena to impedances of inverters and inrush sources connections, and presence of shunt elements between their connections.

Several propositions are given to avoid this problem:

- choosing the measurement frequency in the range of more than $150 \mathrm{~Hz}$ up to a frequency far enough from the first ripple control frequency,

- choosing a longer data window for signal processing (3 or 4 periods),

- choosing a higher number of points for impedance result averaging, and

- skipping a short time interval in measurement after the first high value of impedance is noticed (around $0.5 \mathrm{~s}$ ). Those measurement values can be used for other protections (which have to react quicker). Antiislanding protection can skip them, and it can still have sufficient time to react (within $5 \mathrm{~s}$ ).

\section{Acknowledgements}

This research has been performed within the framework of the IOP-EMVT research program 'Power Quality and EMC' that is supported financially by SenterNovem. SenterNovem is an agency of the Dutch Ministry of Economic Affairs.

\section{References}

[1] W.Bower and M.Ropp, "Evaluation of islanding detection methods for photovoltaic utility interactive power systems", Report IAE PVPS T5-09, March 2002

[2] F. Bertling and S. Soter, "A novel converter integrable impedance measuring method for islanding detection in grids with widespread use of decentral generation", in Proc. SPEEDAM 2006, pp. 503-507

[3] F. De Mango, M. Liserre and A. D. Aquila, "Overview of Anti-Islanding Algorithms for PV Systems. Part II: Active Methods", in Proc. EPEPEMC 2006, pp. 1884-1889
[4] A. V. Timbus, R. Teodorescu, F. Blaabjerg and U. Borup, "Online grid impedance measurement suitable for multiple PV inverters running in parallel", APEC 06, Dallas, 2006

[5] S. Bhattacharyya, Z. Wang, J. F. G. Cobben, J. M. A. Myrzik and W.L. Kling, "Analysis of Power Quality Performance of The Dutch Medium And Low Voltage Grids", ICHP 2008, Wollongong, 2008

[6] Dual Channel FFT Analysis Part I, Technical review No1 -1984, Brüel \& Kjær, 1984. 